\title{
Lower Bone Mineral Can Be a Risk for an Enlarging Bone Marrow Lesion: A Longitudinal Cohort Study of Japanese Women Without Radiographic Knee Osteoarthritis
}

\section{Daisuke Chiba ( $\sim$ dachiba@hirosaki-u.ac.jp )}

Hirosaki University Graduate School of Medicine

\section{Eiji Sasaki}

Hirosaki University Graduate School of Medicine

\section{Seiya Ota}

Hirosaki University Graduate School of Medicine

\section{Tetsushi Oyama}

Hirosaki University Graduate School of Medicine

\section{Yuka Kimura}

Hirosaki University Graduate School of Medicine

\section{Shigeyuki Nakaji}

Hirosaki University Graduate School of Medicine

\section{Yasuyuki Ishibashi}

Hirosaki University Graduate School of Medicine

\section{Research Article}

Keywords: Knee osteoarthritis, Bone marrow lesion, Bone mineral density

Posted Date: February 8th, 2022

DOI: https://doi.org/10.21203/rs.3.rs-1329362/v1

License: (c) (1) This work is licensed under a Creative Commons Attribution 4.0 International License. Read Full License 


\section{Abstract}

Background: Although previous cross-sectional studies have clarified that lower bone mineral density (BMD) is associated with more severe bone marrow lesions (BMLs), its longitudinal relationship remains unclear. The aim of this study was to elucidate the longitudinal relationship between BMD at baseline $(\mathrm{BL})$ and $\mathrm{BML}$ change during a two-year follow-up period (2YFU).

Methods: A total of 78 female participants (Mean age: $54.9 \pm 9.6$ and BMI: $21.6 \pm 3.0 \mathrm{~kg} / \mathrm{m}^{2}$ ) were eligible. All the participants underwent 1.5T magnetic resonance imaging, and fat-suppressed T2weighted images were acquired. Based on coronal and sagittal images, the maximum BML area (BMLa) was calculated by tracing the BML border. The mean value of coronal and sagittal BMLa was also calculated. The change in mean BMLa was defined using the following formula: [BMLa of 2YFU] $[B M L a$ of $B L]=\triangle B M L a$. If $\triangle B M L a$ was positive, it was defined as enlarged; if $\triangle B M L a$ was negative, it was defined as regressed. Dual-energy X-ray absorptiometry was performed to measure the non-dominant side BMD at one-third of the distal radius. Young adult mean [YAM (\%)] of the BMD was used for statistical analysis. Linear regression analysis was conducted with $\triangle B M L a$ as the dependent variable and YAM as the independent variable, adjusted for age, BMI, pain scale, and BMLa at BL. A receiver operating characteristic curve was drawn for the YAM to predict the prevalence of BML enlargement or regression. Logistic regression analysis was conducted with BML enlargement prevalence or regression as the dependent variable, and with the cut-off of YAM as the independent variable. Statistical significance was defined as $P \leq 0.05$.

Results: During the 2YFU, 12 (15.4\%) patients had enlarged BMLa, 26 (33.3\%) participants showed regressing BMLa, and 40 (51.3\%) patients remained stable. The YAM was significantly associated with $\triangle B M L a$, according to the linear regression model $(B:-1.459, b:-0.375, P=0.046)$. The best predictor of the $B M L$ enlargement risk was $85 \%$ YAM; this cut-off was significant in predicting the prevalence of $B M L$ enlargement ( $\mathrm{B}: 2.126$, odds ratio: 8.383, $\mathrm{P}=0.025)$, according to the adjusted logistic regression model.

Conclusion: Lower BMD could predict BML enlargement during a two-year follow-up period.

\section{Background}

The bone-cartilage junction is an important structure for maintaining the homeostasis of the knee joint because it not only supports cartilage tissue as the basis but also metabolic exchange between the cartilage and bone tissue.[1-3] Previous basic studies have reported that the dysfunction of bonecartilage junction, especially subchondral bone tissue, is associated with the initiation of cartilage degeneration to develop knee osteoarthritis (KOA).[1-3] In fact, using magnetic resonance imaging (MRI) findings, several clinical and epidemiological studies have elaborated the relationship between knee symptoms, cartilage degeneration, and subchondral bone damage based on the finding of bone marrow lesions (BMLs) in a population of KOA.[4] Bone marrow lesion is described as an alteration of periarticular bone marrow signal intensity with high signal intensity on fluid-sensitive sequences 
[T2/proton density with fat suppression and short tau inversion recovery (STIR)], which include bone marrow necrosis, bone marrow fibrosis, and microcracks or microbleeding within the subchondral region. $[4,5]$ Cross-sectionally, a larger size of BML was associated with greater knee pain[6-8]; moreover, the change in BML was longitudinally associated with the development or reduction of knee pain.[8,9] Several previous cohort studies have clarified that the existence of BML demonstrated a higher odds ratio of decreasing articular cartilage volume and developing or progressing KOA.[8,10-16] Therefore, controlling the subchondral bone damage in the early phase of KOA has the potential to prevent further deterioration of osteoarthritic changes and OA-related knee symptoms.

In more recent basic studies, the microstructural alterations of subchondral bone are distinct, comparing the early and late stages of KOA. $[1,3]$ In early $O A$, thinner and more porous subchondral bone plates are observed during initial cartilage degeneration.[3] Accordingly, systemic bone fragility derived from osteoporosis is thought to aggravate or mitigate subchondral bone damage of the knee joint. Unfortunately, little is available on the longitudinal data that focuses on the alteration of subchondral bone damage in accordance with systemic bone fragility, while a cross-sectional study has already demonstrated that lower systemic bone mineral density (BMD) is associated with more severe BML grade.[17,18] The cause-effect relationship between systemic fragility and alteration of subchondral bone damage remains unclear. Therefore, the current study aimed to evaluate the longitudinal relationship between the systemic BMD at baseline and the change in subchondral bone damage during two-year follow-up period in a cohort of Japanese women.

\section{Methods}

A total of 1073 volunteers (441 men and 632 women) out of approximately 11,000 eligible people who lived in the Iwaki area of Hirosaki city, participated in the Iwaki Health Promotion Project in 2017 [baseline $(\mathrm{BL})$ ]. Bilateral knee plain radiographs were evaluated according to the Kellgren-Lawrence grade (KLG). [19] A KLG of $\geq 2$ was defined as definitive radiographic KOA. For inclusion criteria, all participants had a KLG of 0 or 1 in the right knee. Additionally, participants with a history of rheumatoid arthritis, knee surgery, and knee trauma were excluded from this study (Figure 1). The ethics committee of Hirosaki University Graduate School of Medicine approved this study, and all participants provided written informed consent before inclusion.

\section{Evaluation of knee symptoms}

To evaluate knee symptoms, all participants answered the knee injury and osteoarthritis outcome scores (KOOS), the most frequently used tool that represents patient-based outcome scores.[20,21] The KOOS consists of 42 knee-related items, and each item is scored from 0 to 4 . Summed scores in each of the four subscales [symptom, pain, activities of daily living (ADL), and QOL] were converted to 100 points, as the best knee condition.

Additionally, based on the classification criteria of "early knee osteoarthritis,"[22] the participants in this study were define as symptomatic or asymptomatic group. The symptomatic group satisfied the 
following criteria: One is that patient-based questionnaires (KOOS) - 2 of the following were required to score "positive" (i.e., $\leq 85 \%$ ): pain (nine items), symptoms (seven items), ADL (short version, seven items), and knee-related quality of life (QOL) (four items). The second consists of clinical examination-at least one of the following needs to be present: joint line tenderness or knee crepitus. The third is radiographs: KLG glade 0 or 1 at the standing, fixed flexion, and weight-bearing positions.

\section{Evaluation of bone mineral density at the distal radius}

Dual-energy X-ray absorptiometry was performed to measure forearm BMD using a DCS-600EXV (Hitachi Aloka Medical, Tokyo, Japan). The BMD region of interest was measured on the non-dominant side at one-third of the distal radius. If there was a history of previous fracture, the dominant side was measured as a substitute for BMD. Since this study was conducted as a part of a community-based general health check project in a limited space of a public hall, it was difficult to measure the spinal and hip BMD, which requires a large facility to shield radioactive materials. Therefore, the forearm was eligible for the measurement of systemic BMD.[17] Finally, the acquired forearm BMD was converted to the young adult mean value [YAM (\%)] to conduct the current statistical analysis.

\section{Evaluation of BML on MRI}

At the baseline and 2-year follow-up, MRI of the right knee joint was acquired. All the participants were scanned with a rapid extremity coil and a mobile 1.5T magnetic resonance unit (ECHELON RX, Hitachi, Tokyo, Japan). During the MRI scanning, the patients were positioned supine with their knees in full extension. The sagittal and coronal planes of T2-weighted fat-suppressed fast-spin echo were performed to evaluate the BML (repetition time 5,000 ms; echo time $80 \mathrm{~ms}$; field of view $16 \mathrm{~cm} ; 288 \times 288$ matrix; and slice thickness of $3 \mathrm{~mm}$ with a gap of $1.0 \mathrm{~mm}$ between the slices). The acquired MRI data were compiled as Digital Imaging and Communications in Medicine (DICOM) files and transferred to an independent computer workstation using the software program OsiriX (Newton Graphic, Inc., Hokkaido, Japan).

On the workstation of Osirix, a single orthopedic surgeon (DC, 14-year career) traced the maximum area of BML to calculate the quantitative value $\left(\mathrm{mm}^{2}\right)$ on sagittal and coronal images; these maximum BMLarea values were averaged and defined as "BML area" (Figure 2). All the MRI measurements were blinded to the information of the participants. Thereafter, the BML area at the 2-year follow-up was subtracted from the $B M L$ area at the $B L$ to determine the amount of change in the $B M L$ area as $\triangle B M L[\triangle B M L=(B M L$ area 2-year follow-up) - (BML area $B L)$ ]. If the participants demonstrated a positive $\triangle B M L$ value, they were defined as an enlargement of the $B M L$ area, and if the participants demonstrated a negative $\triangle B M L$ value, they were defined as regressing the $B M L$ area.

\section{Statistical analysis}

Statistical analysis was performed using the SPSS ver. 24.0 (SPSS Inc., Chicago, IL, USA) in the current study. First, the distribution of all continuous values was examined using the Shapiro-Wilk test. 
According to the distribution results, the mean values of the continuous values were compared using a non-paired t-test or Mann-Whitney $U$ test. Additionally, the differences in mean BML area between baseline and two-year follow-up were compared using the paired t-test or Wilcoxon signed rank test. To evaluate the association between $B M D$ at the $B L$ and $\triangle B M L$, the Pearson's correlation coefficient was calculated. Additionally, to compare the crude and adjusted relationship between the BMD at baseline and $\triangle B M L$, a linear regression analysis was conducted with the $\triangle B M L$ as the dependent variable and with the YAM at baseline as the independent variable, adjusted by the baseline values of age, BMI, KOOS Pain scale, and BML area. Receiver operating characteristic (ROC) curves were drawn to validate the predictive ability of the YAM to diagnose enlargement or regression of the BML area. Based on the results of the ROC curves, we determined the cut-off value of the YAM. Finally, a logistic regression analysis was conducted with the prevalence of BML enlargement or regression as the dependent variable and with the cut-off value of the YAM as the independent variable, adjusted by the baseline values of age, BMI, KOOS Pain scale, and BML area. Statistical significance was defined as P-value of $\leq 0.05$.

\section{Results}

The demographic data of the current study are presented in Table 1. During the two-year follow-up period, 12 (15.4\%) participants enlarged their BML area; 26 (33.3\%) showed regressing of BML area; and 40 (51.3\%) remained stable. At baseline, 63 (80.8\%) participants were assigned to the asymptomatic group and 15 (19.2\%) participants were assigned to the symptomatic group. The mean BML area at BL was $15.9 \pm 31.8 \mathrm{~mm}^{2}$. After the two-year follow-up period, the mean BML area significantly increased to $32.6 \pm$ $62.2 \mathrm{~mm}^{2}(\mathrm{P}=0.021$, Wilcoxon signed rank test $)$ and mean $\triangle B M L$ area was $16.7 \pm 62.8 \mathrm{~mm}^{2}$.

\section{Table 1: Demographic data in the current study}




\begin{tabular}{|c|c|c|c|c|c|c|c|c|c|c|c|}
\hline \multirow[b]{2}{*}{ Baseline } & \multirow[b]{2}{*}{ Age } & \multicolumn{3}{|c|}{ Overall $(\mathrm{N}=78)$} & \multicolumn{3}{|c|}{$\begin{array}{l}\text { Asymptomatic } \\
\text { group }(\mathrm{N}=63)\end{array}$} & \multicolumn{3}{|c|}{$\begin{array}{l}\text { Symptomatic } \\
\text { group }(N=15)\end{array}$} & \multirow{2}{*}{$\begin{array}{l}\text { P-value } \\
1.000\end{array}$} \\
\hline & & 54.9 & \pm & 9.6 & 54.9 & \pm & 10.0 & 55.1 & \pm & 8.2 & \\
\hline & $\mathrm{BMI}$ & 21.6 & \pm & 3.0 & 21.5 & \pm & 3.2 & 22.0 & \pm & 2.1 & 0.203 \\
\hline & $\begin{array}{l}\text { BMD } \\
\left(\mathrm{g} / \mathrm{cm}^{2}\right)\end{array}$ & 0.63 & \pm & 0.12 & 0.62 & \pm & 0.12 & 0.67 & \pm & 0.11 & 0.156 \\
\hline & YAM (\%) & 91.1 & \pm & 16.2 & 90.0 & \pm & 16.8 & 95.3 & \pm & 12.9 & 0.256 \\
\hline & $\begin{array}{l}\text { KOOS } \\
\text { Symptom }\end{array}$ & 91.0 & \pm & 13.1 & 95.4 & \pm & 5.3 & 72.6 & \pm & 19.3 & $<0.001^{*}$ \\
\hline & KOOS Pain & 92.9 & \pm & 12.4 & 97.8 & \pm & 4.1 & 72.0 & \pm & 14.0 & $<0.001^{*}$ \\
\hline & KOOS QOL & 84.5 & \pm & 21.2 & 92.6 & \pm & 11.4 & 50.8 & \pm & 19.7 & $<0.001^{*}$ \\
\hline & KOOS ADL & 97.0 & \pm & 7.1 & 99.6 & \pm & 1.2 & 86.1 & \pm & 10.5 & $<0.001^{*}$ \\
\hline & $\begin{array}{l}\text { BML area } \\
\left(\mathrm{mm}^{2}\right)\end{array}$ & 15.9 & \pm & 31.8 & 13.9 & \pm & 28.0 & 23.8 & \pm & 44.9 & 0.525 \\
\hline
\end{tabular}

$\begin{array}{lllllllllllll}\begin{array}{l}\text { 2-year } \\ \text { follow-up }\end{array} & \begin{array}{l}\mathrm{BML} \text { area } \\ \left(\mathrm{mm}^{2}\right)\end{array} & 32.6 & \pm & 62.2^{\dagger} & 30.4 & \pm & 62.6^{\dagger} & 41.6 & \pm & 61.7 & 0.252 \\ & \begin{array}{l}\Delta \mathrm{BML} \text { area } \\ \left(\mathrm{mm}^{2}\right)\end{array} & 16.7 & \pm & 62.8 & 16.5 & \pm & 61.6 & 17.7 & \pm & 70.2 & 0.854\end{array}$

${ }^{*} \mathrm{P} \leq 0.05$, Mann-Whitney $\mathrm{U}$ test between asymptomatic and symptomatic group; ${ }^{\dagger} \mathrm{P} \leq 0.05$, Wilcoxon signed rank test between baseline and 2-year follow-up.

$\mathrm{BMD}$, bone mineral density; $\mathrm{BML}$, bone marrow lesion; KOOS, knee injury and osteoarthritis outcome score; YAM, young adult mean; ADL, activities of daily living; QOL, quality of life

Overall, the YAM of forearm BMD at BL was negatively associated with the $\triangle B M L$ area. $(r=-0.275$, $\mathrm{P}=0.015$, Pearson's correlation coefficient); this association was more evident in the symptomatic group ( $r$ $=-0.539, P=0.038$, Pearson's correlation coefficient, Figure 3 ). According to the linear regression analysis, the YAM was significantly associated with the $\triangle B M L$ area in the crude and adjusted models (Table 2).

Table 2: Linear regression analysis to evaluate the relationship between the young adult mean of bone mineral density and change of BML area 


\begin{tabular}{lllllllll}
$\begin{array}{l}\text { Dependent } \\
\text { variable }\end{array}$ & $\begin{array}{l}\text { Independent } \\
\text { variable }\end{array}$ & & $\mathrm{B}$ & $\mathrm{b}$ & $\mathrm{P}$ & \multicolumn{2}{c}{$95 \% \mathrm{Cl}$} & \\
\hline$\Delta \mathrm{BML}$ area & YAM of BMD & Crude & - & - & 0.015 & - & - & - \\
& & & 1.071 & 0.275 & & 1.925 & 0.216 \\
\hline & & Adjusted & - & - & 0.046 & - & - & - \\
& & & 1.459 & 0.375 & & 2.891 & 0.026
\end{tabular}

Statistical analysis: Linear regression analysis; B, regression coefficient; $b$, standard regression coefficient; P, P-value; Cl: confidence interval; BMD, bone mineral density; BML, bone marrow lesion; YAM, young adult mean.

The ROC curves demonstrated that the YAM of BMD significantly predicted the enlargement of the BML area (AUC: $0.776,95 \% \mathrm{Cl}: 0.664-0.887, \mathrm{P}<0.001)$; on the contrary, the YAM was insignificant in predicting the regressing of BML area. [Area under curve (AUC): 0.578, $P=0.391$, see Additional file 1) Based on the ROC curve, we could determine the optimal cut-off of YAM as $85 \%$, and this cut-off value was significantly associated with the prevalence of BML enlargement (Adjusted B: 2.126; Odds ratio: 8.383; $\mathrm{P}=0.025 ; 95 \% \mathrm{Cl}: 1.300-54.058$, Table 3).

\section{Table 3: Logistic regression analysis to evaluate the relationship between the YAM of bone mineral density at the baseline and the change of BML area}

\begin{tabular}{|c|c|c|c|c|c|c|c|}
\hline Dependent variable & $\begin{array}{l}\text { Independent } \\
\text { variable }\end{array}$ & & B & OR & $\mathrm{P}$ & $95 \% \mathrm{Cl}$ & \\
\hline \multirow[t]{2}{*}{$\begin{array}{l}\text { Prevalence of BML } \\
\text { enlargement }\end{array}$} & YAM of $85 \%$ & Crude & 2.246 & 9.450 & $<0.001$ & 3.205 & - $\quad 27.863$ \\
\hline & & Adjusted & 2.126 & 8.383 & 0.025 & 1.300 & - $\quad 54.058$ \\
\hline
\end{tabular}

Statistical analysis: logistic regression analysis; $B$, regression coefficient; BML, bone marrow lesion; OR, odds ratio; P, P-value; YAM, Young adult mean; $\mathrm{Cl}, 95 \%$ confidence interval

\section{Discussion}

In a Japanese female cohort without radiographic KOA, we evaluated the longitudinal relationship between the BMD of the distal radius at baseline and change in BML area during a 2-year follow-up. As for the most important finding of this study, converting the BMD to \%YAM value, linear regression analysis clarified that the YAM at baseline was negatively associated with the change in BML area. Based on the ROC curves, the lower YAM was significant in predicting the risk of enlarging the BML area, while this value was not significant in predicting the risk of regressing the BML area. Finally, logistic regression analysis in the current study clarified that a cut-off YAM value of $\leq 85 \%$ could significantly predict the risk of enlarging the BML area. 
Previous epidemiological studies have already discussed the relationship between systemic BMD and incidence or progression of radiographic KOA. In particular, several previous studies clarified how systemic BMDs of the lumbar spine and proximal femur were associated with the incidence of radiographic KOA.[23-29] For participants without definitive radiographic KOA, such as KLG 0 or 1, the majority of previous cohort studies have clarified that higher systemic BMD had increased the risk of incidence of radiographic KOA, compared to lower systemic BMD.[23-29] In other words, those with lower systemic BMD were less likely to develop radiographic KOA than those with higher systemic BMD. Therefore, the current results conflict with those of previous cohort studies. As for the disadvantages of previous epidemiological studies, three points should be considered. First, both male and female populations were included in the analyses. Middle-aged and elderly female patients who are likely to develop KOA are in a period of pre-, peri-, and post-menopause. Accordingly, there is a deficiency of female hormones due to follicular dysfunction; in particular, reducing the secretion of estrogen, which directly suppresses the activity of osteoclasts, overcomes the bone formation derived from osteoblasts.[30,31] Additionally, perimenopausal females are at risk of oxidative stress to induce osteoclast-dominant bone remodeling.[32,33] In short, the epidemiological study of KOA, which considers the relationship between bone metabolism and subchondral bone damage, should divide males and females based on sex-hormone differences to regulate bone metabolism. Second, previous studies reported by Kamil et al.[25] and Bergink et al.[26,27] provided us with demographic data, according to the BMD quartile. Notably, those with a higher quartile of systemic BMD demonstrated a higher BMI compared to those with a lower quartile of systemic BMD. This finding has a potential confounding of higher systemic BMD in which knee articular cartilage is in danger of mechanical stress due to its higher BMI. Additionally, previous evidence has shown that those with higher BMD were associated with higher physical activity; therefore, weight-bearing site BMD, such as the spine and proximal femur are likely to be higher.[34-38] Those with higher physical activity have the potential to stress the articular cartilage of the knee joint to develop KOA. As for the unique point of this study, we evaluated the BMD of the non-weight-bearing site and forearm. Previous female-cohort studies reported that physical activity had little effect on the change in distal-radius BMD in cross-sectional[39,40] and longitudinal designs.[41] Therefore, the BMD of the non-weight-bearing site may demonstrate a novel specificity of bone metabolism, compared to that of the weight-bearing sites, such as the spine and proximal femoral BMD. Future research would have the potential to clarify the detailed mechanism of non-weight-bearing BMD on articular cartilage degeneration of the knee joint. Third, only plain radiographs of the knee joint were evaluated in previous cohort studies that elucidated the relationship between systemic BMD and the incidence of KOA. Basically, the osteoarthritic changes on plain radiographs of the knee joint are relatively slow. In the general population with KOA, Mazzuka et al. reported that the medial femorotibial joint space narrows to less than $0.10 \mathrm{~mm} /$ year[42], and a more recent systematic review concluded that the annual rate of medial femorotibial joint space narrowing was $0.13 \pm 0.15 \mathrm{~mm} / y e a r$.[43] Additionally, it is difficult to detect a more detailed mechanism of periarticular bone damage, such as BML; MRI has an advantage of evaluating real-time bony pathology in comparison to plain radiography. Notably, the current data could longitudinally evaluate the alteration of BML in the female cohort with pre-radiographic KOA. Moreover, we could clarify the longitudinal relationship between the alteration of periarticular bone 
damage during the 2-year follow-up and the baseline BMD of the forearm. In line with more recent basic studies $[1,3]$ to elucidate the pathological mechanism of subchondral bone porosity in the early stage of $\mathrm{KOA}$, the current epidemiological study warns of the risk of enlarging BML in the non-radiographic-KOA female population with lower systemic BMD, indicating systemic bone fragility. Therefore, maintaining higher systemic BMD in females in the early stage of KOA has the potential to prevent the enlargement of further BML in the knee joint, which is a potential risk factor for cartilage degeneration and KOA.[8,10-16]

Subchondral bone damage, such as BML is one of the pathological factors that emerged, not only in cartilage degeneration $[10,11,14]$ but also in patients with both early and late stages of KOA[6-9]. Previous epidemiological studies have reported that knee symptoms in patients with KOA worsened in accordance with the severity of BML.[6,9] Interestingly, previous research in the recent decade has argued that knee symptoms derived from OA were significantly improved by administering bisphosphonate, which suppresses bone absorption and restores the systemic BMD in patients with osteoporosis.[44,45] In particular, it is notable that administering bisphosphonate reduced BML area concomitant with the improvement of knee pain[46-51]; moreover, in a more recent epidemiological study, Hayes et al. reported that bisphosphonate demonstrated the possibility of a disease-modifying drug in terms of preventing radiographic $O A$ progression in a population with $K L G<2$. For middle-aged women, menopause linked to the hormonal reduction of estrogen causes a systemic bone turnover hyper; accordingly, they are likely to have a risk of fragility or porosity in the subchondral bone of the knee joint. Therefore, the current data would support that bisphosphonate can improve the knee symptoms derived from subchondral bone damage by alternating the systemic and periarticular bone microenvironment. Especially in middle-aged or elderly women without definitive radiographic KOA, preventing lower systemic BMD has the potential to reduce the risk of periarticular bone damage in the knee joint, which contributes to further development of KOA.

Another interesting finding of this study is that the optimal cut-off value to determine the risk of enlarging the BML area was $85 \%$ YAM of BMD, which is substantially higher than the $70 \%$ of YAM to determine the risk of fracture relative to osteoporosis.[52,53] In other words, the threshold of systemic bone strength to induce periarticular bone damage would be higher than that required to induce osteoporotic fracture. Accordingly, future studies should focus on the relationship between systemic bone strength and periarticular bone damage of the knee joint to distinguish the outcome to induce periarticular bone damage from that to induce osteoporotic fracture. The pathologies of bone metabolism between the periarticular damage of the knee joint and osteoporotic fracture are likely to be different.

The current study had several limitations. First, the participants in this study consisted of only women with non-radiographic KOA. Therefore, the relationship between BMD and the amount of change in the $\mathrm{BML}$ area is still unclear in the male population, and definitive radiographic KOA. Second, the sample size of the symptomatic group was relatively small, and it was difficult to conduct further multi-variable analysis for this group. Third, BMD was measured at the forearm, which is a non-weight-bearing site. Although the spinal and proximal femoral BMDs are the gold standard for evaluating systemic bone 
fragility, it was difficult to measure the spinal and proximal femoral BMD, which requires a large facility to shield radioactive materials, since this study was conducted as a part of a community-based general health check project in a limited space of a public hall. Therefore, the forearm was eligible for the substitutional measurement of systemic BMD in this study.[17] However, the BMD of the non-weightbearing site would show the different pathological meaning of the weight-bearing site, such as the spinal or proximal femur in terms of the alteration of periarticular bone damage. Despite these limitations, the current data can be variable for patients with early-stage KOA who have the potential to progress to definitive KOA. For these patients, lower systemic BMD is the risk of enlarging the area of BML, and maintaining a higher systemic BMD would prevent further deterioration of periarticular bone damage, which is linked to further osteoarthritic change and knee symptoms. Future studies will clarify the more detailed mechanisms of systemic BMD to control periarticular bone damage in the knee joint.

\section{Conclusion}

Based on a Japanese female cohort with non-radiographic KOA, this study evaluated the longitudinal relationship between the BMD of the distal radius at baseline and the change in BML area during the 2year follow-up. Converting the BMD value to the YAM, linear regression analysis clarified that the YAM at baseline was negatively associated with the change in BML area. Additionally, the YAM was significant in predicting the risk of enlarging the BML area, and logistic regression analysis clarified that the cut-off value of YAM ( $\leq 85 \%)$ could predict the risk of enlarging the BML area. For patients with early-stage KOA, lower systemic $B M D$ is the risk of enlarging the area of $B M L$, and maintaining a higher systemic $B M D$ would prevent further periarticular bone damage, which is linked to further osteoarthritic change and knee symptoms.

\section{List Of Abbreviations}

$\mathrm{BMD}$, bone mineral density; $\mathrm{BML}$, bone marrow lesion; KOOS, knee injury and osteoarthritis outcome score; YAM, young adult mean; $A D L$, activities of daily living; $Q O L$, quality of life; $B M I$, body mass index; KLG, Kellgren-Lawrence grade; $B L$, baseline; MRI, magnetic resonance imaging; ROC, receiver operating characteristic; 2YFU, two-year follow-up; KOA, knee osteoarthritis

\section{Declarations}

Ethics approval and consent to participate: The ethics committee of Hirosaki University Graduate School of Medicine approved this study, and all participants provided written informed consent before inclusion.

Consent for publication: Not applicable.

Availability of data and materials: The current database is available only for those who request to testify the validity of this study and acquire the consent from the corresponding author.

Competing interests: The authors declare that they have no competing interests. 
Funding: The present study was supported in part by JST COI Grant Number JPMJCE1302, a Grant-in-Aid from the Japanese Society for the Promotion of Science (Nos. 21500676, 18K16606, 18K09091), the Health Labor Sciences Research Grant, a JOA-Subsidized Science Project Research from the Japanese Orthopedic Association (2018-4), and the Grant of Japan Orthopedics and Traumatology Research Foundation (No. 421).

Author contributions: DC drafted the manuscript and chiefly organized the design of this study and statistical analysis. ES chiefly organized the data collection of knee symptoms, plain radiograph, and MRI. SO and TO conducted collecting the actual data from the current study participants. YK conducted the review of manuscript. SN conducted the entire organization of the Iwaki Health Promotion Project and the final approval to submit the manuscript. YI reviewed the manuscript and conducted the final approval to submit the manuscript.

Acknowledgement: The authors are grateful to the medical staff who supported the conduct of the Iwaki Health Promotion Project, as well as Editage for English language editing.

\section{References}

1. Li G, Yin J, Gao J, Cheng TS, Pavlos NJ, Zhang C, et al. Subchondral bone in osteoarthritis: insight into risk factors and microstructural changes. Arthritis Res Ther. 2013;15:223.

2. Yuan XL, Meng HY, Wang YC, Peng J, Guo QY, Wang AY, et al. Bone-cartilage interface crosstalk in osteoarthritis: potential pathways and future therapeutic strategies. Osteoarthritis Cartilage. 2014;22:1077-89.

3. Hu W, Chen Y, Dou C, Dong S. Microenvironment in subchondral bone: predominant regulator for the treatment of osteoarthritis. Ann Rheum Dis. 2021;80:413-22.

4. Roemer FW, Frobell R, Hunter DJ, Crema MD, Fischer W, Bohndorf K, et al. MRI-detected subchondral bone marrow signal alterations of the knee joint: terminology, imaging appearance, relevance and radiological differential diagnosis. Osteoarthritis Cartilage. 2009;17:1115-31.

5. Kon E, Ronga M, Filardo G, Farr J, Madry H, Milano G, et al. Bone marrow lesions and subchondral bone pathology of the knee. Knee Surg Sports Traumatology Arthrosc. 2016;24:1797-814.

6. Aso K, Shahtaheri SM, McWilliams DF, Walsh DA. Association of subchondral bone marrow lesion localization with weight-bearing pain in people with knee osteoarthritis: data from the Osteoarthritis Initiative. Arthritis Res Ther. 2021;23:35.

7. Ota S, Sasaki E, Sasaki S, Chiba D, Kimura Y, Yamamoto Y, et al. Relationship between abnormalities detected by magnetic resonance imaging and knee symptoms in early knee osteoarthritis. Sci Rep. 2021;11:15179.

8. Driban JB, Price LL, Lo GH, Pang J, Hunter DJ, Miller E, et al. Evaluation of bone marrow lesion volume as a knee osteoarthritis biomarker-longitudinal relationships with pain and structural changes: data from the Osteoarthritis Initiative. Arthritis Res Ther. 2013;15:R112. 
9. Felson DT, Niu J, Guermazi A, Roemer F, Aliabadi P, Clancy M, et al. Correlation of the development of knee pain with enlarging bone marrow lesions on magnetic resonance imaging. Arthritis Rheum. 2007;56:2986-92.

10. Cai G, Aitken D, Laslett LL, Hill C, Wluka AE, March L, et al. The association between change in bone marrow lesion size and change in tibiofemoral cartilage volume and knee symptoms. Rheumatology. 2021;60:2791-800.

11. Teichtahl AJ, Cicuttini FM, Abram F, Wang Y, Pelletier J-P, Dodin P, et al. Meniscal extrusion and bone marrow lesions are associated with incident and progressive knee osteoarthritis. Osteoarthritis Cartilage. 2017;25:1076-83.

12. Sharma L, Nevitt M, Hochberg M, Guermazi A, Roemer FW, Crema M, et al. Clinical significance of worsening versus stable preradiographic MRI lesions in a cohort study of persons at higher risk for knee osteoarthritis. Ann Rheum Dis. 2016;75:1630.

13. Roemer FW, Kwoh CK, Hannon MJ, Hunter DJ, Eckstein F, Fujii T, et al. What Comes First? Multitissue Involvement Leading to Radiographic Osteoarthritis: Magnetic Resonance Imaging-Based Trajectory Analysis Over Four Years in the Osteoarthritis Initiative. Arthritis Rheumatol. 2015;67:2085-96.

14. Sharma L, Chmiel JS, Almagor O, Dunlop D, Guermazi A, Bathon JM, et al. Significance of Preradiographic Magnetic Resonance Imaging Lesions in Persons at Increased Risk of Knee Osteoarthritis. Arthritis Rheumatol. 2014;66:1811-9.

15. Roemer FW, Guermazi A, Javaid MK, Lynch JA, Niu J, Zhang Y, et al. Change in MRI-detected subchondral bone marrow lesions is associated with cartilage loss: the MOST Study. A longitudinal multicentre study of knee osteoarthritis. Ann Rheum Dis. 2009;68:1461.

16. Hunter DJ, Zhang Y, Niu J, Goggins J, Amin S, LaValley MP, et al. Increase in bone marrow lesions associated with cartilage loss: A longitudinal magnetic resonance imaging study of knee osteoarthritis. Arthritis Rheum. 2006;54:1529-35.

17. Ota S, Chiba D, Sasaki E, Kumagai G, Yamamoto Y, Nakaji S, et al. Symptomatic bone marrow lesions induced by reduced bone mineral density in middle-aged women: a cross-sectional Japanese population study. Arthritis Res Ther. 2019;21:113.

18. Zhu Q, Xu J, Wang K, Cai J, Wu J, Ren J, et al. Associations between systemic bone mineral density, knee cartilage defects and bone marrow lesions in patients with knee osteoarthritis. Int J Rheum Dis. 2018;21:1202-10.

19. Kellgren JH, Lawrence JS. Radiological Assessment of Osteo-Arthrosis. Ann Rheum Dis. 1957;16:494-502.

20. Roos EM, Roos HP, Lohmander LS, Ekdahl C, Beynnon BD. Knee Injury and Osteoarthritis Outcome Score (KOOS)-Development of a Self-Administered Outcome Measure. J Orthop Sport Phys. 1998;28:88-96.

21. Nakamura N, Takeuchi R, Sawaguchi T, Ishikawa H, Saito T, Goldhahn S. Cross-cultural adaptation and validation of the Japanese Knee Injury and Osteoarthritis Outcome Score (KOOS). J Orthop Sci. 2011;16:516-23. 
22. Luyten FP, Bierma-Zeinstra S, Dell'Accio F, Kraus VB, Nakata K, Sekiya I, et al. Toward classification criteria for early osteoarthritis of the knee. Semin Arthritis Rheu. 2018;47:457-63.

23. Sowers M, Lachance L, Jamadar D, Hochberg MC, Hollis B, Crutchfield M, et al. The associations of bone mineral density and bone turnover markers with osteoarthritis of the hand and knee in pre- and perimenopausal women. Arthritis Rheum. 1999;42:483-9.

24. Hart DJ, Cronin C, Daniels M, Worthy T, Doyle DV, Spector TD. The relationship of bone density and fracture to incident and progressive radiographic osteoarthritis of the knee: The Chingford study. Arthritis Rheum. 2002;46:92-9.

25. Barbour KE, Murphy LB, Helmick CG, Hootman JM, Renner JB, Jordan JM. Bone Mineral Density and the Risk of Hip and Knee Osteoarthritis: The Johnston County Osteoarthritis Project. Arthrit Care Res. 2017;69:1863-70.

26. Bergink AP, Rivadeneira F, Bierma-Zeinstra SM, Zillikens MC, Ikram MA, Uitterlinden AG, et al. Are Bone Mineral Density and Fractures Related to the Incidence and Progression of Radiographic Osteoarthritis of the Knee, Hip, and Hand in Elderly Men and Women? The Rotterdam Study. Arthritis Rheum 2019;71:361-9.

27. Bergink AP, Uitterlinden AG, Leeuwen JPTMV, Hofman A, Verhaar JAN, Pols HAP. Bone mineral density and vertebral fracture history are associated with incident and progressive radiographic knee osteoarthritis in elderly men and women: The Rotterdam Study. Bone. 2005;37:446-56.

28. Nevitt MC, Zhang Y, Javaid MK, Neogi T, Curtis JR, Niu J, et al. High systemic bone mineral density increases the risk of incident knee $O A$ and joint space narrowing, but not radiographic progression of existing knee OA: the MOST study. Ann Rheum Dis. 2010;69:163.

29. Hochberg MC, Lethbridge-Cejku M, Tobin JD. Bone mineral density and osteoarthritis: Data from the Baltimore Longitudinal Study of Aging11Supported in part by grants from the Arthritis Foundation, Maryland Chapter and the National Institute of Arthritis, Musculoskeletal and Skin Diseases. Osteoarthritis Cartilage. 2004;12:45-8.

30. Zaidi M, Turner CH, Canalis E, Pacifici R, Sun L, Iqbal J, et al. Bone loss or lost bone: Rationale and recommendations for the diagnosis and treatment of early postmenopausal bone loss. Curr Osteoporos Rep. 2009;7:118-26.

31. Zebaze RM, Ghasem-Zadeh A, Bohte A, luliano-Burns S, Mirams M, Price RI, et al. Intracortical remodeling and porosity in the distal radius and post-mortem femurs of women: a cross-sectional study. Lancet. 2010;375:1729-36.

32. Manolagas SC. From Estrogen-Centric to Aging and Oxidative Stress: A Revised Perspective of the Pathogenesis of Osteoporosis. Endocr Rev. 2010;31:266-300.

33. Kousteni S. FoxOs: Unifying Links Between Oxidative Stress and Skeletal Homeostasis. Curr Osteoporos Rep. 2011;9:60.

34. Perez-Lasierra JL, Casajus JA, González-Agüero A, Moreno-Franco B. Association of physical activity levels and prevalence of major degenerative diseases: Evidence from the national health and nutrition examination survey (NHANES) 1999-2018. Exp Gerontol. 2022;158:111656. 
35. Kim YA, Lee Y, Lee JH, Seo JH. Effects of physical activity on bone mineral density in older adults: Korea National Health and Nutrition Examination Survey, 2008-2011. Arch Osteoporos. 2019;14:103.

36. Chastin SFM, Mandrichenko O, Helbostadt JL, Skelton DA. Associations between objectivelymeasured sedentary behaviour and physical activity with bone mineral density in adults and older adults, the NHANES study. Bone. 2014;64:254-62.

37. Taaffe DR, Robinson TL, Snow CM, Marcus R. High-Impact Exercise Promotes Bone Gain in WellTrained Female Athletes. J Bone Miner Res. 1997;12:255-60.

38. Shimegi S, Yanagita M, Okano H, Yamada M, Fukui H, Fukumura Y, et al. Physical Exercise Increases Bone Mineral Density in Postmenopausal Women. Endocr J. 1994;41:49-56.

39. Haapasalo H, Kannus P, Sievänen H, Pasanen M, Uusi-Rasi K, Heinonen A, et al. Effect of Long-Term Unilateral Activity on Bone Mineral Density of Female Junior Tennis Players. J Bone Miner Res. 1998;13:310-9.

40. Adami S, Gatti D, Braga V, Bianchini D, Rossini M. Site-Specific Effects of Strength Training on Bone Structure and Geometry of Ultradistal Radius in Postmenopausal Women. J Bone Miner Res. 1999;14:120-4.

41. Simões D, Craveiro V, Santos MP, Camões M, Pires B, Ramos E. The effect of impact exercise on bone mineral density: A longitudinal study on non-athlete adolescents. Bone. 2021;153:116151.

42. Mazzuca SA, Brandt KD, Katz BP. Is conventional radiography suitable for evaluation of a diseasemodifying drug in patients with knee osteoarthritis? Osteoarthritis Cartilage. 1997;5:217-26.

43. Emrani PS, Katz JN, Kessler CL, Reichmann WM, Wright EA, McAlindon TE. et al. Joint space narrowing and Kellgren-Lawrence progression in knee osteoarthritis: an analytic literature synthesis. Osteoarthritis Cartilage. 2008;16:873-82.

44. Hagino $\mathrm{H}$, Kishimoto $\mathrm{H}$, Ohishi $\mathrm{H}$, Horii S, Nakamura T. Efficacy, tolerability and safety of oncemonthly administration of $75 \mathrm{mg}$ risedronate in Japanese patients with involutional osteoporosis: A comparison with a $2.5 \mathrm{mg}$ once-daily dosage regimen. Bone. 2014;59:44-52.

45. Bone HG, Hosking D, Devogelaer J-P, Tucci JR, Emkey RD, Tonino RP, et al. Ten Years' Experience with Alendronate for Osteoporosis in Postmenopausal Women. New Engl J Medicine 2004;350:1189-99.

46. Ballal P, Sury M, Lu N, Duryea J, Zhang Y, Ratzlaff C, et al. The relation of oral bisphosphonates to bone marrow lesion volume among women with osteoarthritis. Osteoarthritis Cartilage 2020;28:1325-9.

47. Haj-Mirzaian A, Guermazi A, Roemer FW, Bowes MA, Conaghan PG, Demehri S. Bisphosphonates intake and its association with changes of periarticular bone area and three-dimensional shape: data from the Osteoarthritis Initiative (OAI). Osteoarthr Cartilage 2018;26:564-8.

48. Vaysbrot EE, Osani MC, Musetti M-C, McAlindon TE, Bannuru RR. Are bisphosphonates efficacious in knee osteoarthritis? A meta-analysis of randomized controlled trials. Osteoarthritis Cartilage. 2018;26:154-64.

49. Varenna M, Zucchi F, Failoni S, Becciolini A, Berruto M. Intravenous neridronate in the treatment of acute painful knee osteoarthritis: a randomized controlled study. Rheumatology. 2015;54:1826-32. 
50. Laslett LL, Kingsbury SR, Hensor EMA, Bowes MA, Conaghan PG. Effect of bisphosphonate use in patients with symptomatic and radiographic knee osteoarthritis: data from the Osteoarthritis Initiative. Ann Rheum Dis. 2014;73:824-30.

51. Laslett LL, Doré DA, Quinn SJ, Boon P, Ryan E, Winzenberg TM, et al. Zoledronic acid reduces knee pain and bone marrow lesions over 1 year: a randomised controlled trial. Ann Rheum Dis. 2012;71:1322-8.

52. Orimo H, Hayashi Y, Fukunaga M, Sone T, Fujiwara S, Shiraki M, et al. Diagnostic criteria for primary osteoporosis: year 2000 revision. J Bone Miner Metab. 2001;19:331-7.

53. Osteoporosis JS for $B$ and MR and JOSJRC for the R of the DC for P, Soen S, Fukunaga M, Sugimoto T, Sone T, Fujiwara S, et al. Diagnostic criteria for primary osteoporosis: year 2012 revision. J Bone Miner Metab. 2013;31:247-57.

\section{Figures}

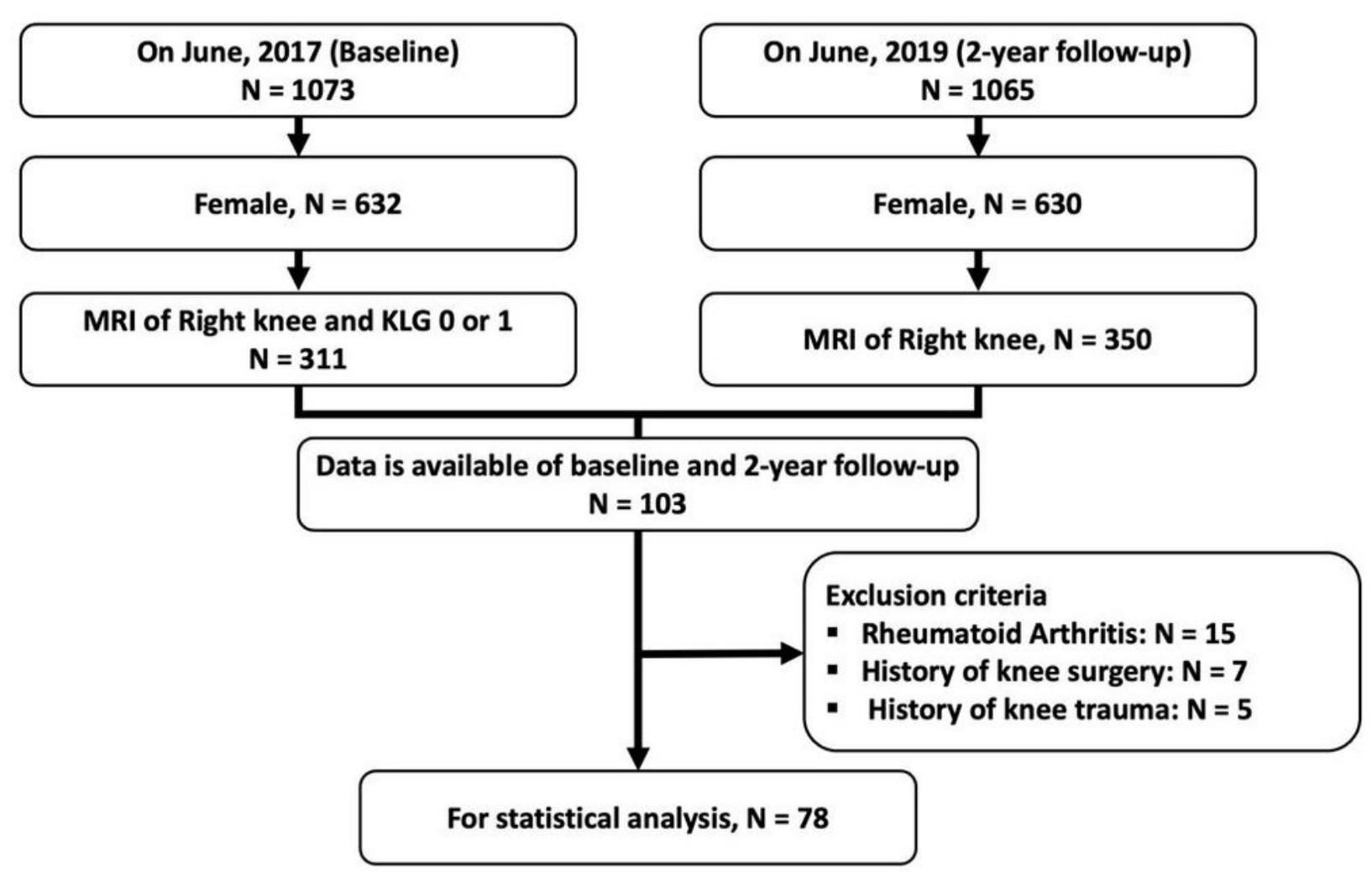

Figure 1

Flow chart showing recruitment of the study participants

KLG: Kellgren-Lawrence grade. 


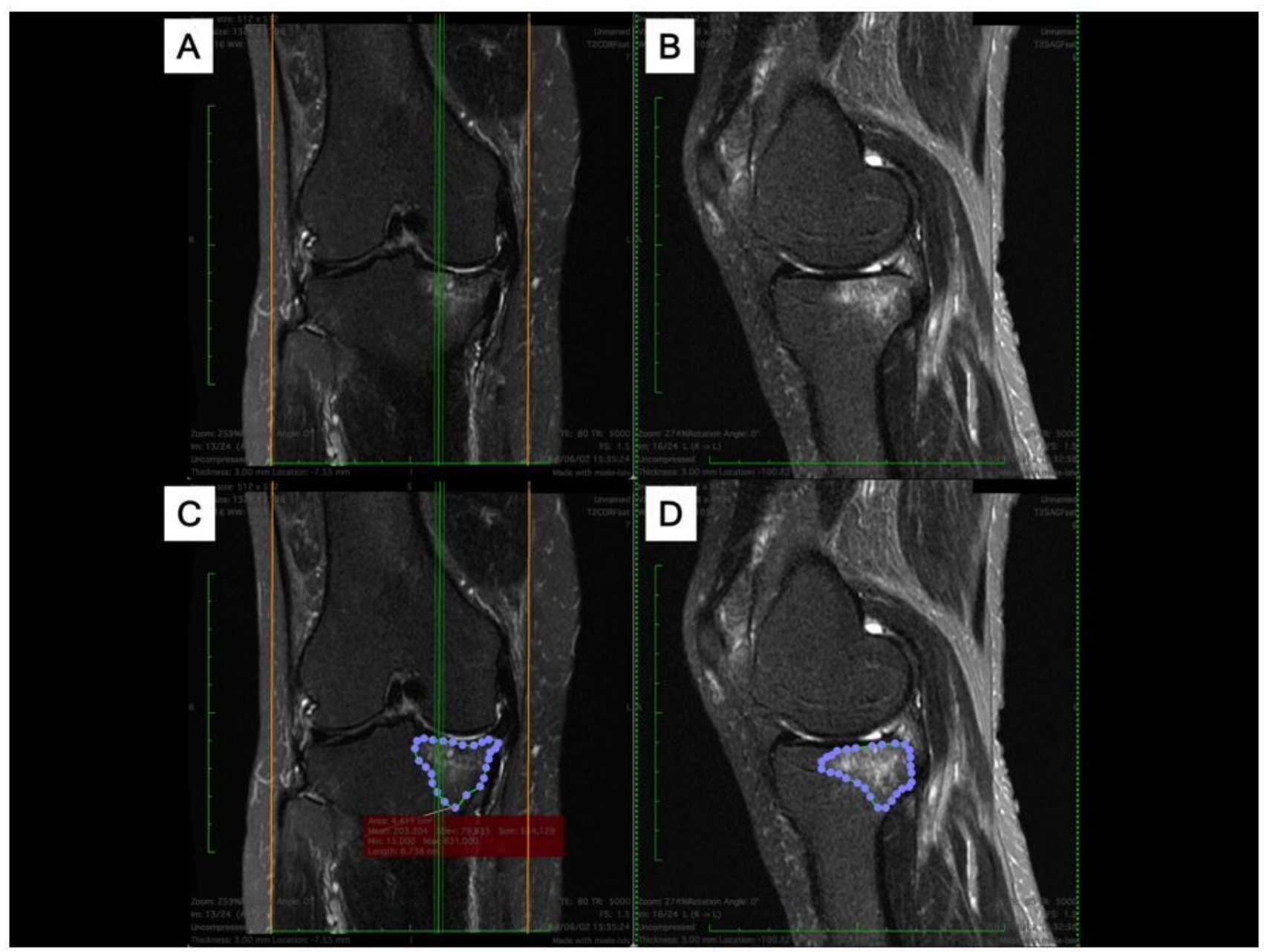

Figure 2

Measuring the area of bone marrow edema on magnetic resonance images

A) T2-weighted fat-suppression coronal image; B) T2-weighted fat-suppression sagittal image; C) and D) Traced area of bone marrow lesion on coronal and sagittal images. 


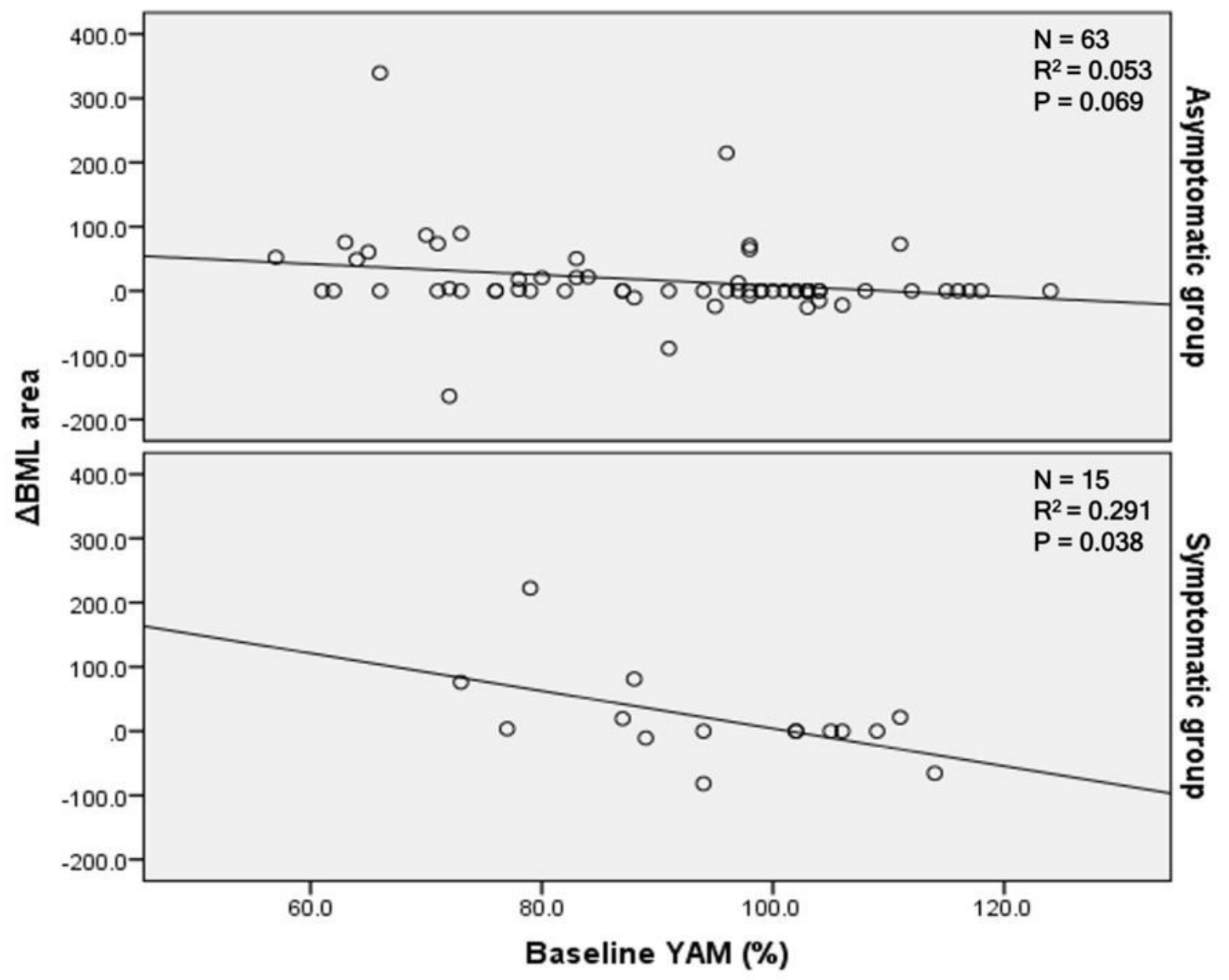

Figure 3

Scattergram to compare the relationship between the young adult mean of bone mineral density at the baseline and $\triangle B M L$ area

$\mathrm{BML}$, bone marrow lesion; $\mathrm{R}^{2}$, coefficient of determination; YAM, young adult mean

\section{Supplementary Files}

This is a list of supplementary files associated with this preprint. Click to download.

- 20220204BMLiAdditionalfile1.docx 